Journal of Engineering and Applied Sciences 14 (4): 1279-1285, 2019

ISSN: 1816-949X

(C) Medwell Journals, 2019

\title{
Hybrid Method for Detection Tumor using Genetic Algorithm and Swarm Optimization after Wavelet Domain Filtering Then uing Marr-Hilerth
}

\author{
Hind Rustum Mohammed and Lamyaa Fahem Katran \\ Department of Computer Science, Faculty of Computer Science and Mathematics, \\ University of Kufa, Kufa, Iraq
}

\begin{abstract}
Brain tumors consist of several types of abnormal growth within the skull and the Central Nervous System (CNS). They result from abnormal and uncontrolled able cell division. Brain tumors are dangerous and when left untreated can result in loss of life. Some tumors, however does not lead to death, especially, the lipomas which are inherently unharmful. The potential risk associated with tumors depends on several factors such as the type of the tumuor its size and location as well as the condition of the tumor. This study proposes the use of pre-processing phase wave image filter size box algorithm for brain tumor imaging as this algorithm provides improved image quality devoid of noise. The suggested method contains three essential steps, the first step involves the use of a Genetic algorithm which has a zero tolerance for probability and flexibility and can also find near-optimal solutions. In the second step an entrained Particle Sswarm Optimization (PSO) technique was used to mechanically determine the mid-clustering the randomly collected data set. The third stage involved the merging of one and two regions of segmentation in the data set using marshalled to detect a tumor in the brain. The noise in the image was filtered using the Laplacian-Gaussian technique before edge detection. The Laplacian-Gaussian technique involves the merging of a Gaussian filtering technique with the Laplacian technique for edge detection. The technique involves three main steps-filtering, improvement and discovery before finally calculating the tumor area using the proposed algorithm.
\end{abstract}

Key words: Magnetic Resonance Imaging (MRI), wavelet domain filtering square magnitude Genetic algorithm, particle swarm optimization, Marr-Hildreth, seed image region, tumor area

\section{INTRODUCTION}

Brain tumor consists of two main types, the benign (non-cancerous) and the malignant (cancerous) tumors. The cancerous brain tumors can develop from any part of the body and slowly spread out to other parts of the human body and the brain too. For this, they are referred to as cancerous or metastatic tumors (Mohammed and Katran, 2018; Singh et al., 2004). A non-cancerous or benign brain tumor consists of a mass of relatively slowly growing cells that settle in any part of the brain. They do not spread from one region to another and if surgically removed do not often relapse. Benign tumors that are not entirely removed during a surgical procedure can grow back but will not affect the other parts of the brain. This behavior of both types of tumor requires close monitoring using scans or treatment with https:// www.nhs.uk/conditions/radiotherapy/pages/introduction aspxeither chemotherapy or radiotherapy. Benign brain tumors are of different types based on the type of brain cells they affect. Some of the common benign brain tumors include the gliomas which affects the glial tissue that provides support to the nerve fibres and cells, the meningioma that affect the meninges that cover the brain surface, the acoustic neuromas https://www.nhs.uk/ conditions/acoustic/neuroma/pages/introduction aspx (also known as vestibular schwannomas) which affect the acoustic nerves, the craniopharyngiomas that affect the base of the brain. They are the most diagnosed form of brain tumor in children, teenagers and adolescents. Others include the haemangioblastoma as which affect the blood vessels of the brain and the pituitary adenomas which affect the pituitary gland. Brain cancer consists of the primary brain tumors which develop in the brain and does not metastasize to other body parts, the secondary tumors which develop from tumors from other parts of the body and metastasis a size to the brain. There are several types of brain tumor (more than 40 major types), grouped into 2 major types. The first type is the benign or slow-growing types which are unlikely to etastasize. Common types of

Corresponding Author: Hind Rustum Mohammed, Department of Computer Science,

Faculty of Computer Science and Mathematics, University of Kufa, Kufa, Iraq 


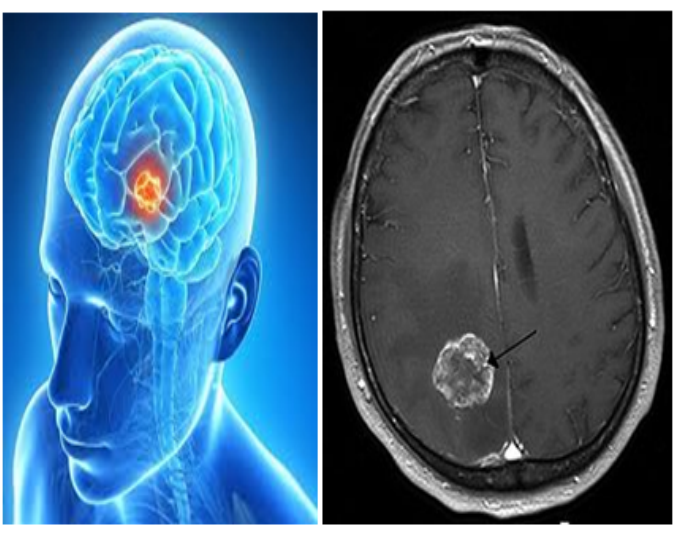

Fig. 1: Image of tumor in brain of human

benign brain tumors include meningiomas, pituitary tumors and neuromas. The second type is the malignant or cancerous brain tumors which can spread from one part of the brain to the other including to the spinal cord. Some examples of the malignant brain tumors include tracy tumors, globalist, oligomer drogliom as and mixed gliomas (Fig. 1).

\section{MATERIALS AND METHODS}

Study outline: The pre-processing step (image noise removal) was performed using a wavelet domain filtering square magnitude image software. Removal of noisy is necessary from the data in order to proceed with further data analysis. In literature, many methods are mentioned for removal of noise. It is generally classified into two categories: denoising in the original signal or image domain (e.g., time or space) and denoising in the transform domain (e.g., Fourier or WT). Before using a merged GA and PSO algorithm for feature selection. Finally, the Marr-Hildreth was used for tumor boundary and area calculation (Fig. 2).

\section{Image processing and feature extraction}

Image pre-processing: The image was pre-processed using wavelet domain filtering square magnitude image processor for noise removal. This method was preferred in this study because the orthogonal wavelet transforms the power of the preferred image signal into a small number of coefficients. Hence, the transformed image consists of a small number of coefficients with high SNR that was kept and many coefficients with low SNR which were discarded. Having discarded the noisy coefficients, the image was reconstructed using the inverse wavelet transform. With this method, the image can be filtered from the noise (Nowak, 1999; Donoho, 1993). The wavelet domain filtering square image algorithm is as follows

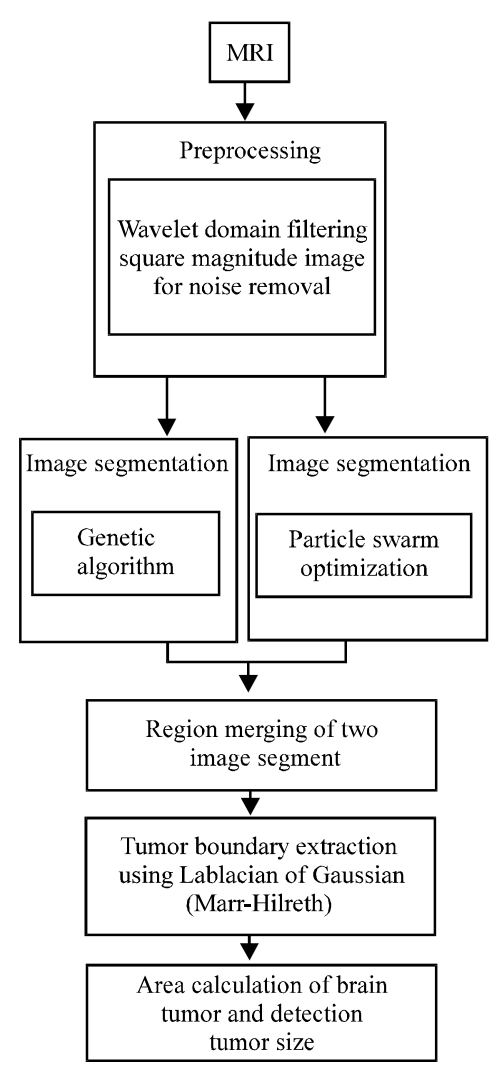

Fig. 2: Block diagram of a purposed system

(Nowak, 1999) compute the J-scale DWT and DWST of the squared magnitude image of $\sigma^{2} \mathrm{I}$. Form estimates of the variances of each wavelet coefficients:

$$
\begin{gathered}
d_{I}, d_{I}=\sum_{m} W_{I}[m] s[m], d_{I}=\sigma_{I} d_{I} \\
\sigma_{I}^{2}=4 \sigma^{2} \operatorname{MAX}\left[\left(\sum_{m} w^{2}[m] x^{2}[m]\right)-\sigma^{2}, \sigma^{2}\right], \sigma^{2} \text { is } 4 \sigma^{4}
\end{gathered}
$$

According to is used todistinguish this $d_{I}$ note that wavelet ccoefficient from the wavelet coefficient corresponding to the magnitude image $\mathrm{x}[\mathrm{m}]$. Underlying discretetime wavelet basic $\mathrm{w}_{\mathrm{I}}^{\mathrm{k}}$ function at scale $\mathrm{K}$ and location I. Filter the wavelet coefficients according to:

$$
\frac{\mathrm{d}_{\mathrm{I}}^{2}}{\mathrm{~d}_{\mathrm{I}}^{2}}-\frac{\mathrm{T}^{2} \sigma_{\mathrm{I}}}{\mathrm{d}_{\mathrm{I}}^{2}}
$$

Remove the basic from the scaling coefficients by subtracting $\mathrm{C}_{\mathrm{I}}$ from each:

$$
\mathrm{C}=2^{(\mathrm{j}+1) \sigma^{2}}
$$


Obtain an estimate of $S^{2}$ by computing the inverse DWT of the filtered waveletand scaling coefficients. Use the pixel-by-pixel square root of the result to get an estimate of $\mathrm{s}$. It should be noted that $\mathrm{d}_{\mathrm{I}}$ was used to differentiate this wavelet coefficient from that was used to differentiate this wavelet coefficient from that of $d_{I}$ which corresponds to the magnitude image:

$$
\mathrm{X}[\mathrm{m}] \mathrm{d}_{\mathrm{I}}=\sum_{\mathrm{m}} \mathrm{w}_{\mathrm{I}}[\mathrm{m}] \mathrm{s}[\mathrm{m}], \mathrm{d}_{\mathrm{I}}=\delta_{\mathrm{I}} \mathrm{d}_{\mathrm{I}}, \mathrm{w}_{\mathrm{I}}^{\mathrm{k}}
$$

De note the underlying discrete time wavelet basic function at scale $\mathrm{k}$ and location.

Feature extraction: This process identifies the feature characteristics that shows the predictable tumor in the brain through the mass of the output image from the image processing software. There are two stages in this processm, the first stage is the segmentation of the image mass using GA. The segmentation technique can be classified into classical algorithms which are mainly based on statistical or mathematical methods, optimization-based techniques and artificial intelligence-based techniques (Dagar and Dahiya, 2016). The classical algorithms comprise characteristic histogram threshold, edge/boundary detection, region extraction or region increasing, recreation, semantic and syntactic move toward. The segmentation techniques are applied AI methods that use artificial neural networks. The GA is mainly used in image segmentation for the modification of the existing parameters in the segmentation algorithm and for segmentation at pixel level (Holland, 1992). GA can determine the optimal number of segmentation result regions and can choose some features such as the heuristic thresholds or the size of the analysis window (Back and Kursawe, 1995; Farmer and Shugars, 2006). The GA is robust and can be applied in different fields due to its basic functionality. Other algorithms in the same class with the GA include Evolutionary Strategy (ES), Genetic Programming (GP) and Evolutionary Programming (EP). A major difference between GA and other conventional optimization methods its ability to use a population of points at the same time in contrast to the single point approach used by the conventional optimization methods. AGA presents the genetic representation of the solution domain as well as a fitness function for the evaluation of the solution domain.

The second stage of the feature selection process is determining the mass of the image using PSO. Digital image segmentation involves the unravelling of a digital image into its various sections. In the image dispensation system it is a complex but necessary task. Image segmentation is performed to present the image in an obvious way. It is usually used to dissipate an image into regions that ideally correspond to different real-world substances. Image segmentation is a risky step towards image and content psychoanalysis. Its result is a collection of segments that converge to form the real image. There are several problems of real globe image segmentation including feature minimization, overall deviation reduction, classifier error rate reduction and connectivity maximization. The PSO was developed by Kenney and Eberhart (Bhattacharyya, 2011; Gaurav and Bansal, 2013). An evolutionary calculation technique after merge by merge area using Marr-Hildreth to the output of merge two phase of segmentation using region merging algorithm.

Genetic algorithm: The consists of the choosing the initial individual population, evaluating the fitness of the individuals in the population and repeat this process until the termination criteria (time limit, sufficient fitness achieved) is met. The subsequent steps include selecting the best-fit individuals for reproduction, breeding new individuals through mutation and crossover operations, evaluating the fitness of the new individuals and replacing the least-fit individuals with the best-fit new individuals.

Image segmentation using GA: As per (Farmer and Shugars, 2006) there are two types of Genetic algorithms for image segmentation-parameter selection which involves the parameters of an existing image segmentation technique is modified using GA to improve its output and pixel-level segmentation which involves the use of GA to perform region labelling. Most methods of image segmentation have parameters that demands to be optimized, hence, the first method is often used (Bhattacharyya, 2011).

Practical Swarm Optimization (PSO): The PSO is a class of swarm-based intelligence techniques used to handle optimization issues. The PSO mimics the flocking behaviour of bird where a group of birds randomly search an area for food and in this area there is just a piece of food being searched. The birds have no idea of the position of the food but they know about its location in the search space. In this situation, the ideal way to locate the food is to either forage or flock. The location of each particle in the swarm is updated following two "best" values-best and g-best with the p-best, each particle keeps track of its coordinates in the search space with respect to the best solution (fitness) it has so far achieved and hence, the name p-best or personal best. The g-best 


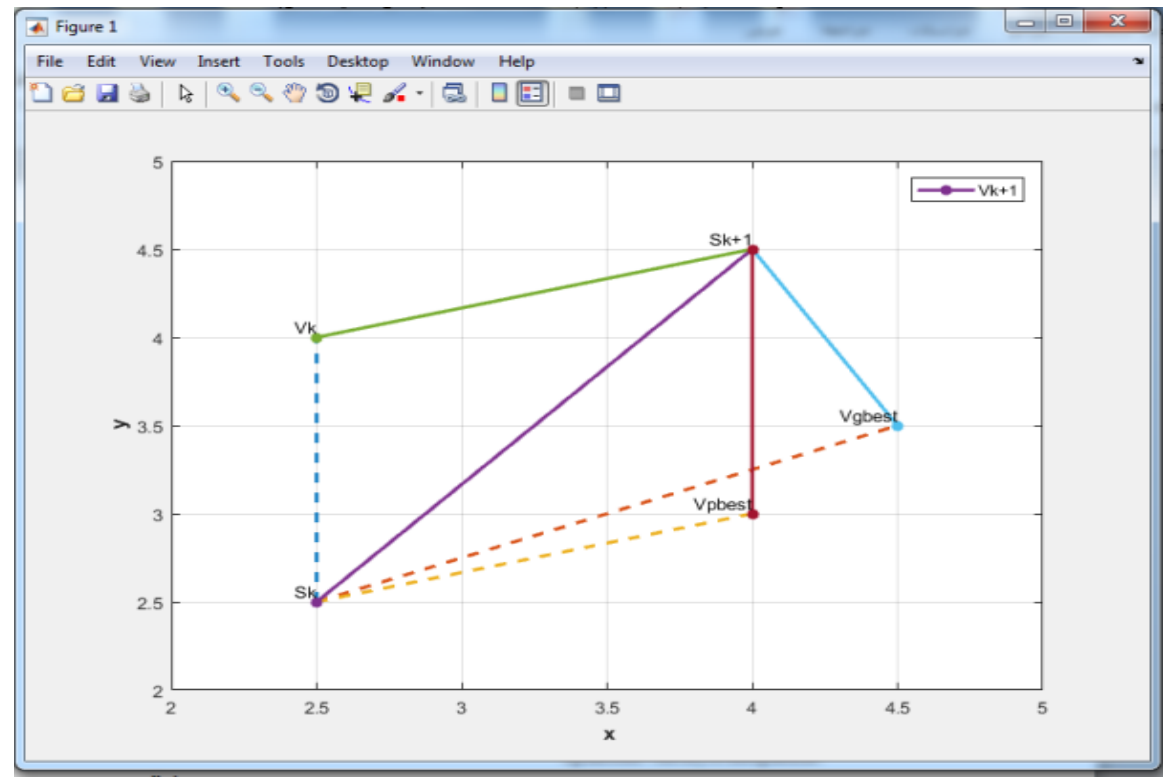

Fig. 3: Concept of modification of a searching point by PSO

is tracked by the PSO as the best value, so far obtained by any particle within the neighbourhood of the particle and hence, the name g-best or global best. The position of each particle is updated using their current positions and velocities the distance between the p-best and the current position as well as the distance between g-best and the current position. Having established the two best values, the velocity and position of the particle are updated using the following Eq. 2 (Bhattacharyya, 2011):

$$
\begin{gathered}
\mathrm{v}[]=\mathrm{v}[]+1^{*} \operatorname{rand}()^{*}(\text { pbes[]-ppresent[] })+ \\
\mathrm{c}_{2}{ }^{*} \text { rand }()^{*}(\text { gbest[]-ppresent[] })
\end{gathered}
$$

Where:

$$
\text { (1) present [] = present []+v[] }
$$

$\mathrm{S}^{\mathrm{K}}=$ Current Searching point

$\mathrm{S}^{\mathrm{K}+1}=$ Modified Searching point

$\mathrm{V}^{\mathrm{K}}=$ Modified Velocity

$\mathrm{V}^{\text {pbest }}=$ Velocity based on pbest

$\mathrm{V}^{\text {gbest }}=$ Velocity based on gbest

where, $v[]$ is the velocity of the particle, present [] is the current particle (solution). rand() is a random number between $(0 ; 1) \cdot c_{1-2}$ are learning factors usuallycl $=\mathrm{c}_{2}=2$. The concept of modification of a searching point by $\mathrm{PSO}$ algorithm is depicted in Fig. 3 (Bhattacharyya, 2011).

In Fig. 3, it shows the concept of modification of PSO. Each particle moves from the current position to the next one according to the present values. Generally, the fitness function is same the objective functions. The local best of other particles in the population should be changed if the present fitness function value is better than the previous. The algorithm of the PSO is as follows (Gaurav and Bansal, 2013):

\section{Alghorithm 1: PSO; Gaurav and Bansal (2013)}

Step 1: Let's represent the number of particles in the swarm with each particle having a position $X_{i}$ and velocity $v_{i}$ in the search space. Le's represent the best-achieved position of particle I and $g$ be the best for the entire swarm. Abasic PSO algorithm is executed thus

Step 2: For each particle $\mathrm{i}=1, \ldots, \mathrm{S}$, perform the following: 1 use a uniformly distributed random vector $\mathrm{xi} \sim \mathrm{U}$ (blo, bup) to initialize the position of the particle where blo and up represent the lower and upper limits of the solution space

2 Initialize the best-known position of the particle to its initial position: $p_{i}$ $\mathrm{x}_{\mathrm{i}}$ If $\left(\mathrm{f}\left(\mathrm{p}_{\mathrm{i}}\right)<\mathrm{f}(\mathrm{g})\right)$

3-Update the best-known position of the swarm pi

4-Initialize the velocity of the particle

Step 3: Repeat the following steps until the termination criteria (such as the number of iterations performed or have found a solution with adequate objective function value) is met

For each particle $i=1, \ldots, S$ do

For eachdimension $\mathrm{d}=1, \ldots, \mathrm{n}$ do

Pick random numbers: $\mathrm{rp} ; \mathrm{rg} \sim \mathrm{U}(0 ; 1)$

Updatethe velocity of theeparticle

Update the position of the particle: $\mathrm{x} \mathrm{x}_{\mathrm{i}}+\mathrm{v}_{\mathrm{i}}$

If $\left(\mathrm{f}\left(\mathrm{x}_{\mathrm{i}}\right)\right.$ If $\left.\left(\mathrm{p}_{\mathrm{i}}\right)\right)$ do

Update the most excellent known position of the particle: $p_{i} x_{i}$

If $\left(f\left(p_{i}\right) 1 f(g)\right)$, update the best-identified position of the swarm: $g p_{i}$

Step 4: $g$ is now the best found solution. The parameters, $\varphi p$ and $\varphi g$ are chosen by the practitioner controlling the legacy and behavior of the PSO

Region merging: An appropriate seed selection is a significant consideration during the segmentation process. The selected seed pixels must (Kansal and Jain, 2015). Be highly similar to its neighbouring pixels. At least one seed pixel must be generated for each expected image region. There should be a connection between the seeds 
of different image regions. In the proposed approach, the occurrence frequency of the seeds after merging was considered during seed selection. For each image, the seeds were computed using the following algorithm 2 .

Algorithm 2; PSO base algorithm:

Step 1: Compute the image grey levels and sort them in ascending order

Step 2: Compute the frequency of the soft image grey levels

Step 3: Assign the first pixel as the first seed point

Step 4: Merge the pixels to get an improved seed point. If the merging conditions are met, replace the seed point by the mean of the merged pixels Sum up the frequencies of the pixel and the seed point, assign the position of the seed point to be equal to the initial seed point, otherwise, assign the pixel as a new seed point. Repeat step 4 until all the pixels are unmerged Step 5: Sort the final seed points based on their occurrence frequency in descending order. Thus, the final seed points achieved are used for image segmentation in the seeded region growing algorithm

Marr-Hildreth: Edge detection is a basic image processing and computer vision technique. It detects the areas in a digital image where there are sharp changes in the image brightness or where there are discontinuities (Tandan et al., 2014). It is used for noise filtration before edge discovery. The Marr-Hildreth method is a combination of the Gaussian filtering and Laplacian edge detection techniques.

Definition of the components: Laplacian is a measure of an image's second spatial derivative and is a useful tool in detecting abrupt changes in images. Gaussian smoothing is performed in image edging before Laplacian to annul the noise effect. The operations are linear and can be interchanged. Gaussian smoothing is a unique form of weighted smoothing where the smoothing kernel coefficients are derived from a Gaussian distribution. The 2-DLaplacian of Gaussian function centred on zero and with Gaussian standard deviation has the form a variation of the value of the standard deviation controls the amount of smoothing.

Calculate area of tumor: Apply the algorithm of find area (Suman et al., 2017) of tumor image as shown in Fig. 4 and 5 .

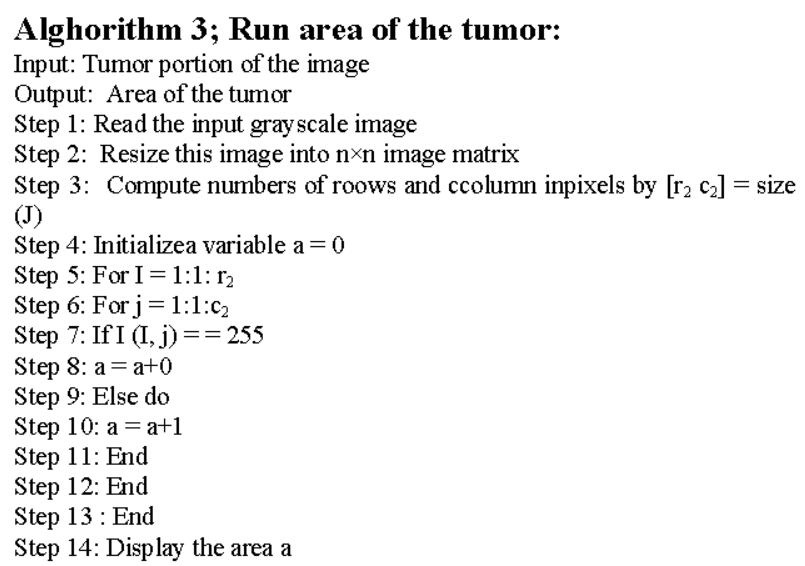

Step 3: Compute numbers of roows and ccolumn inpixels by $\left[\mathrm{r}_{2} \mathrm{c}_{2}\right]=$ size (J)

Step 4: Initializea variable $\mathrm{a}=0$

Step 5: For $\mathrm{I}=1: 1: \mathrm{r}_{2}$

Step 6: For $\mathrm{j}=1: 1: \mathrm{c}_{2}$

Step 7: If I $(\mathrm{I}, \mathrm{j})==255$

Step 8: $\mathrm{a}=\mathrm{a}+0$

Step 9: Else do

Step 10: $\mathrm{a}=\mathrm{a}+1$

Step 11: End

Step 12: End

Step $13:$ End

Step 14: Display the area a

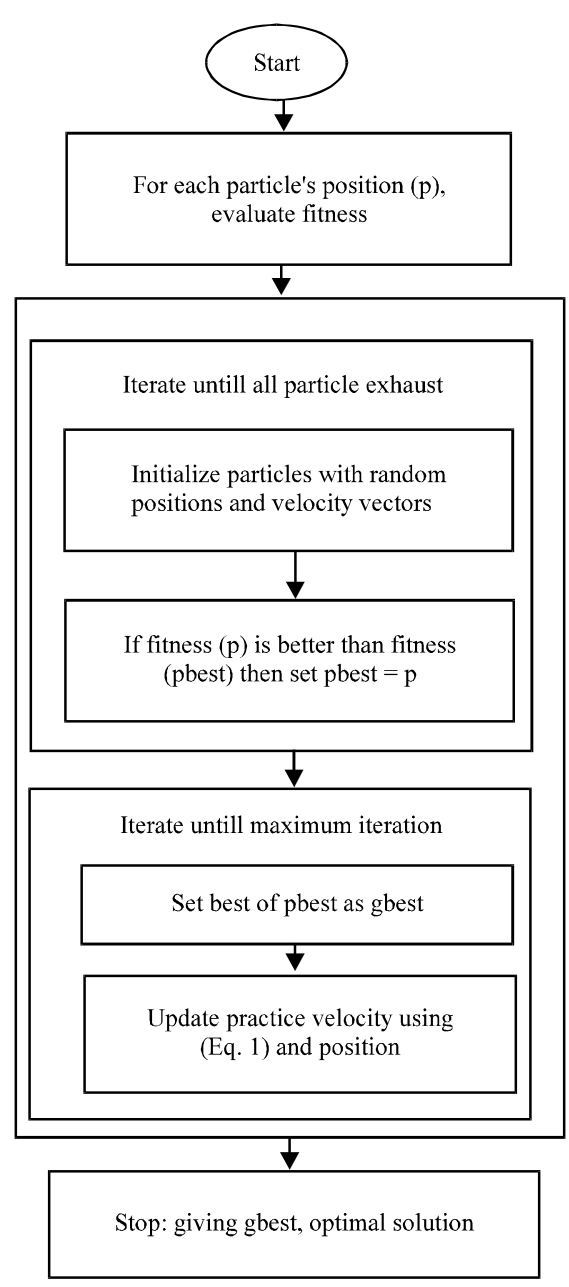

Fig. 4: Flowchart of the PSO base algorithm

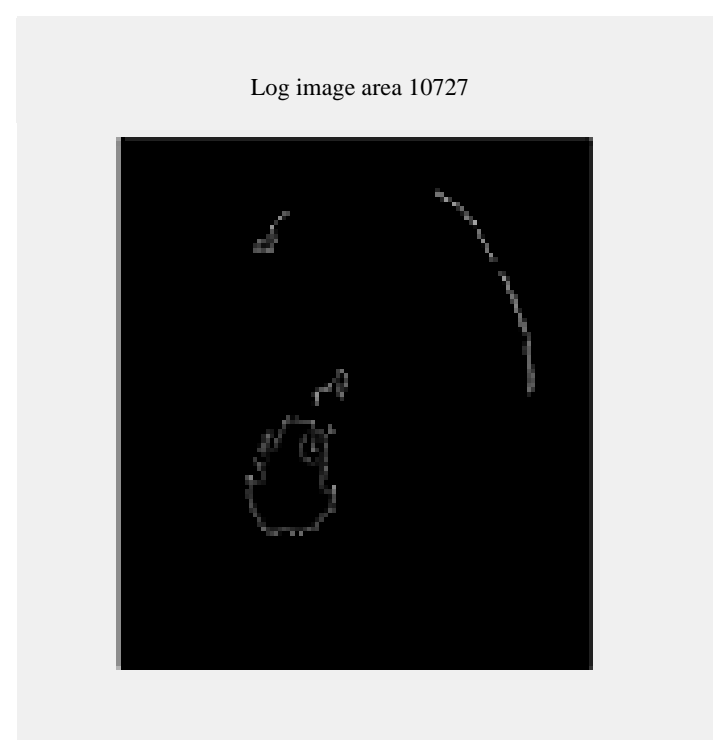

Fig. 5: Run area of the tumor 


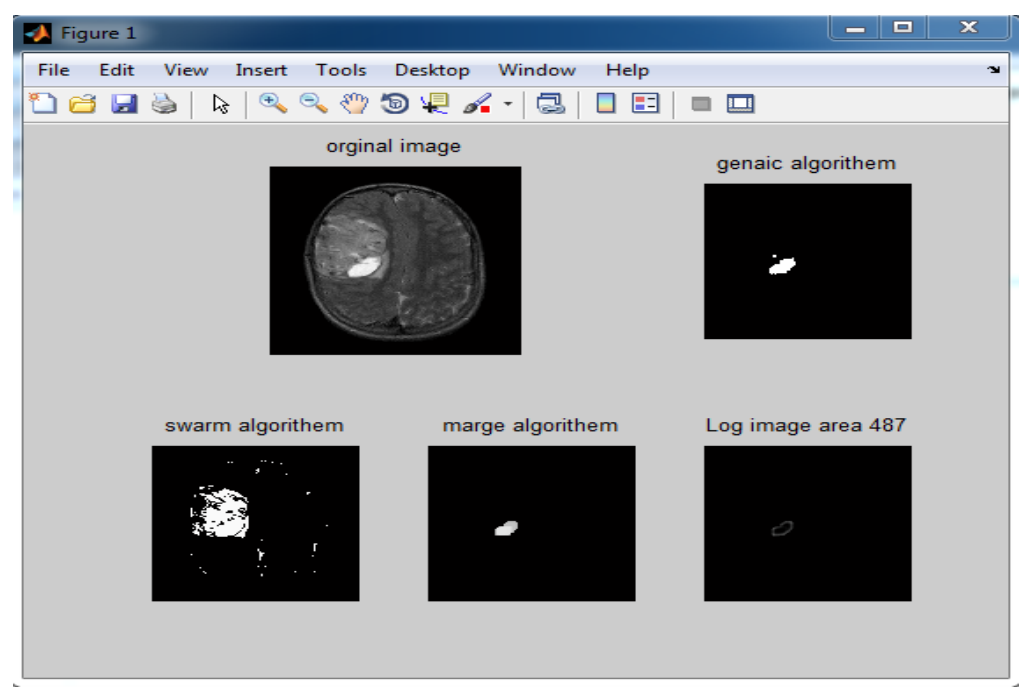

Fig. 6: Run of all work

\begin{tabular}{|c|c|c|c|c|}
\hline $\mathrm{TP}$ & $\mathrm{TN}$ & $\mathrm{FP}$ & $\mathrm{FN}$ & Total \\
\hline 3 & 25 & 2 & 0 & 30 \\
\hline Metrics & Values & \multicolumn{2}{|c|}{ LAW } \\
\hline Accuracy & 0.93333 & \multicolumn{2}{|c|}{$(\mathrm{TP}+\mathrm{TN}) /$ Total } \\
\hline $\begin{array}{c}\text { Misclassification } \\
\text { error }\end{array}$ & 0.06 & \multicolumn{2}{|c|}{$(\mathrm{FP}+\mathrm{FN}) /$ Total } \\
\hline Sensitive P & 1 & $\mathrm{TP} / \mathrm{TP}+\mathrm{FN}$ \\
\hline Sensitive N & 0.9259 & $\mathrm{TN} / \mathrm{TN}+\mathrm{FP}$ \\
\hline Precision (PPV) & 0.6 & \multicolumn{2}{|c|}{$\mathrm{TP} / \mathrm{TP}+\mathrm{FP}$} \\
\hline NPV & 1 & \multicolumn{2}{|c|}{$\mathrm{TN} / \mathrm{TN}+\mathrm{FN}$} \\
\hline
\end{tabular}

Fig. 7: Calculate the metrics for run program

\section{RESULTS AND DISCUSSION}

Images set: The presentation of brain tumor was assessed using a merged GA and PSO. The dataset was acquired through magnetic resonance imaging where this search using 30 image for testing the detect tumor in magnetic resonance imaging.

Evaluation performance factors: The of the proposed technique's performance was evaluated using a set of examination data. Based on this, the output image for detecting a tumor in the brain is shown in Fig. 6 calculate metrics to perform the program of this thesis by calculating accuracy that shows in Fig. 7 to compare with future or old thesis to see a good performance of the thesis. PPV is positive predictive value NPV is Negative

Predictive Value. TP is genuinely positive, TN is a real negative, FP is False Positive and FN is a False Negative.

\section{CONCLUSION}

Genetic algorithm has many compensations in getting the optimized reply. It was shown to be the majority influential optimization method in a large space. The Genetic algorithm allows to carry out healthy. The outcome of the optimizations depend on the genetic material indoctrination system and participation of genetic employee as well as on the strength function. However, the fineness of image segmentation can be better by choosing the parameters in an optimized method. While PSO is the usual method of calculating and give a number of habits to decide real-world harms more professionally and rapidly with correctness.

\section{RECOMMENDATION}

In feature, research can use one of the previous methods of segment remember in study with other develop method of the segment and deploy to detect a tumor in the brain.

\section{REFERENCES}

Back, T. and F. Kursawe, 1995. Evolutionary Algorithms for Fuzzy Logic: A Brief Overview. In: Fuzzy Logic and Soft Computing, Bouchon-Meunier, B., R.R. Yager and A.Z. Lotfi (Eds.). World Scientific, Singapore, New Jersey, London, Hong Kong, ISBN:9789810223458, pp: 3-10. 
Bhattacharyya, S., 2011. A brief survey of color image preprocessing and segmentation techniques. J. Pattern Recognit. Res., 6: 120-129.

Dagar, N.S. and P.K. Dahiya, 2016. Soft computing techniques for edge detection problem: A State-of-the-art review. Intl. J. Comput. Appl., 136: 28-34.

Donoho, D.L., 1993. Unconditional bases are optimal bases for data compression and for statistical estimation. Appl. Comput. Harmon. Anal., 1: 100-115.

Farmer, M.E. and D. Shugars, 2006. Application of genetic algorithms for Wrapper-based image segmentation and classification. Proceedings of the IEEE International Congress on Evolutionary Computation CEC, July 16-21, 2006, IEEE, Vancouver, Canada, pp: 1300-1307.

Gaurav, K. and H. Bansal, 2013. Particle Swarm Optimization (PSO) technique for image enhancement. Intl. J. Electron. Commun. Technol., 4: 117-119.

Holland, J.H., 1992. Adaptation in Natural and Artificial Systems: An Introductory Analysis with Applications to Biology, Control and Artificial Intelligence. 2nd Edn., The MIT Press, USA., ISBN-10: 0262581116, pp: 228.
Kansal, S. and P. Jain, 2015. Automatic seed selection algorithm for image segmentation using region growing. Intl. J. Adv. Eng. Technol., 8: 362-367.

Mohammed, H.R. and L.F. Katran, 2018. Hybrid method for detection of brain tumor using fuzzy C-mean clustering and discrete curve let transform. Intl. J. Appl. Eng. Res., 13: 1670-1674.

Nowak, R.D., 1999. Wavelet-based Rician noise removal for magnetic resonance imaging. Image Process. Trans., 8: 1408-1408.

Singh, S.K., C. Hawkins, I.D. Clarke, J.A. Squire and J. Bayani et al., 2004. Identification of human brain tumour initiating cells. Nature, 432: 396-401.

Suman, D., N.N. Siddiqui, N. Kriti and S.P. Tamang, 2017. Detection and area calculation of brain tumour from MRI images using MATLAB. Intl. J. Comput. Eng. Res. Trends, 4: 37-40.

Tandan, A., R. Raja and Y. Chouhan, 2014. Image segmentation based on particle swarm optimization technique. Intl. J. Sci. Eng. Technol. Res., 3: 189-202. 